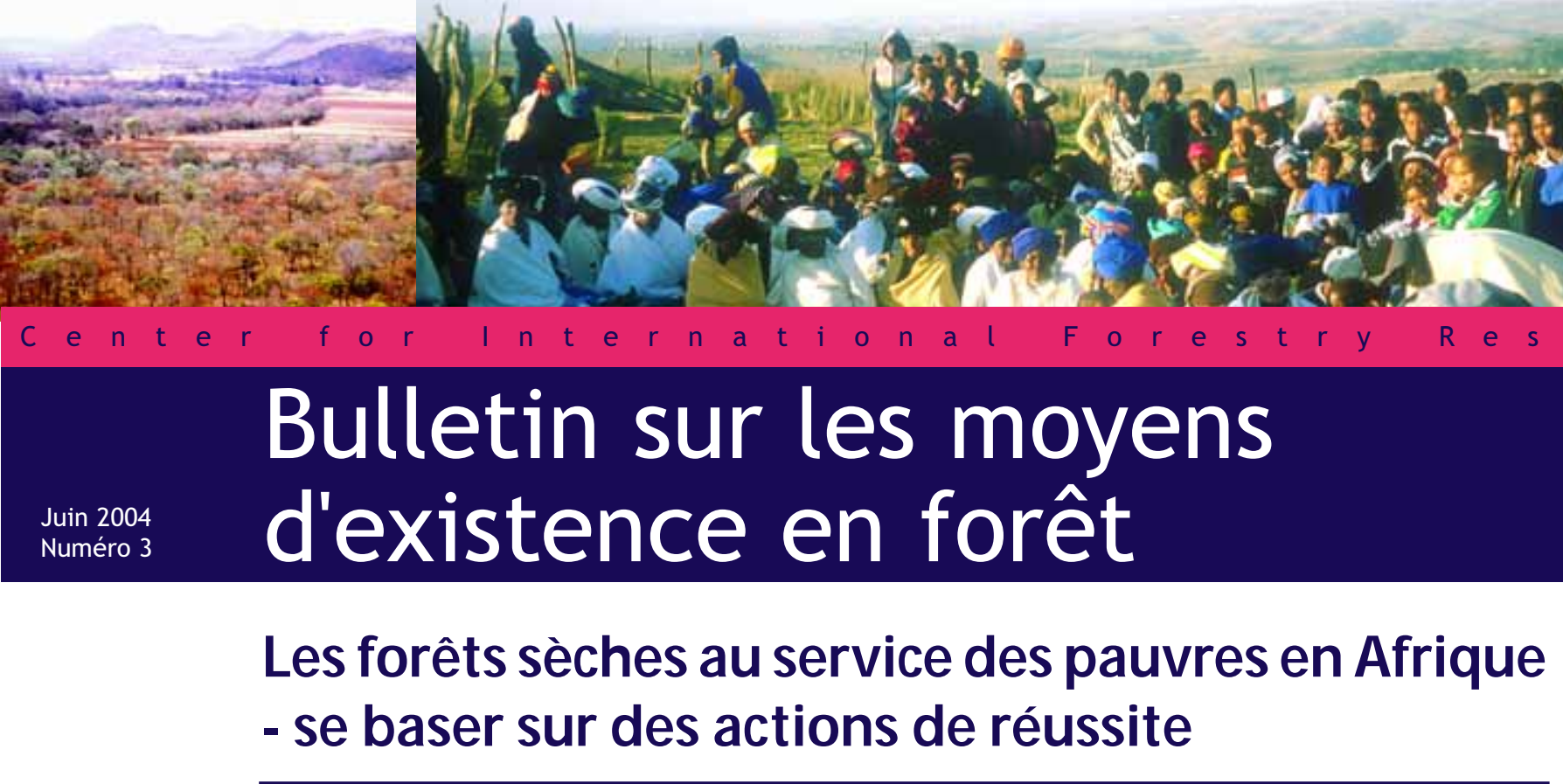

\title{
Leçons apprises
}

L'éradication de la pauvreté représente aujourd'hui le plus grand défi mondial auquel le monde doit faire face et constitue une condition préalable nécessaire pour un développement durable. Il est nécessaire d'entreprendre des actions audacieuses afin d'atteindre les buts de réduction de la pauvreté. Dans ces informations brèves sur les moyens de subsistance nous présentons quelques faits de succès qui montrent comment la gestion durable des ressources forestières a conduit à des impacts positifs en matière de moyens de subsistance. Ceci nous permet d'apprendre quatre leçons principales :

- Les produits forestiers peuvent augmenter les moyens de subsistance des populations, mais la promotion de leur valeur exige des changements au niveau de la forme et du lieu des produits ainsi que de la durée de leur livraison sur le marché.

- L'entreprenariat est important. Les gens qui ont réussi ont eu la confiance de saisir l'initiative quand les opportunités se sont présentées.

- Il est important de s'organiser ; quand les gens peuvent s'organiser ils ont des opportunités d'échanger des informations, d'apprendre des expériences des autres, de partager des ressources et d'entreprendre des activités conjointes. Ils peuvent s'exprimer davantage.

- L'assistance extérieure peut faire la différence en aidant les gens à améliorer la qualité de leurs produits, à avoir accès à la technologie et aux marchés ou à surmonter d'autres barrières à l'entrée.

\section{L'or liquide - assurer les moyens de subsistance et l'économie zambienne}

"C'est le deuxième approvisionnement en miel", dit Pious Makeche en enlevant le rayon de miel du seau devant les balances de l'acheteur, "et c'est de l'or liquide pur qui me permettra d'acheter une bicyclette pour mon enfant pour aller à l'école". Le second approvisionnement a lieu entre mars et mai quand les grands arbres mutondo de la Province du nord-ouest de Zambie commencent à fleurir à la fin de la saison pluvieuse. Le mutondo qui est l'un des arbres les plus courants des vastes régions boisées du miombo de l'Afrique Centrale, produit du nectar de haute qualité que les abeilles africaines transforment en miel embaumé légèrement ambré. Les producteurs récoltent le miel dans des ruches suspendues à des branches d'arbres à travers toute la forêt. Les rayons de miel sont mis dans des seaux et vendus aux coopératives des producteurs et à des sociétés privées qui le raffinent pour ensuite l'exporter en Europe. De grandes étendues de terres dans le miombo sont des zones biologiques certifiées qui assurent au consommateur un produit naturel et propre et garantissent un bon marché au producteur.

Au cours de la dernière décennie, lorsque les conditions économiques se sont aggravées pour la plupart des communautés rurales vivant dans cette région reculée de la Zambie, le commerce du miel a joué un rôle clé dans la vie économique des populations en apportant des revenus aux ménages pauvres. Un kilogramme de miel apporte à un ménage environ 40 cents US presque la moitié du revenu quotidien moyen. La demande de miel s'accroît. Des nouvelles technologies de production telles que l'utilisation des ruches kenyanes améliorées encouragent des milliers de femmes à produire elles-mêmes le miel. Des acheteurs pleins de discernement font continuellement la promotion des pratiques de récolte plus durables, par exemple en n'achetant pas le miel trop clair obtenu de l'état sauvage.

Mais il reste encore beaucoup à faire pour permettre à plus de ménages ruraux de bénéficier de ces avantages. Les producteurs et les agents de vulgarisation doivent connaître les techniques qui permettent d'augmenter la production et la qualité. Les acheteurs ont besoin de bénéficier de prêts à faible taux. Les infrastructures de commercialisation et de traitement du miel nécessitent une attention 
particulière surtout en matière d'accès aux technologies avancées pour l'examen et le traitement du miel afin de répondre aux exigences standards des acheteurs occidentaux. "Ca sera dur", dit Evelyne Zimba, une acheteuse du secteur privé socialement responsable, "mais si nous pérseverons, nous réussirons". Les producteurs, les transformateurs, les commerçants, le gouvernement - nous tous qui travaillons ensemble...Un jour, la Zambie figurera sur la carte du monde comme le pays produisant la meilleure qualité du miel jamais connu".

L'Alliance Zambienne pour la Population et l'Environnement (ZAPE), grâce à l'appui financier de l'IFAD et de l'Asdi, aide le Département de la Foresterie Zambienne à développer une politique "d'élevage" des abeilles qui soutient tous les acteurs impliqués dans la commercialisation du miel, en aidant la Zambie à répondre aux quotas d'exportation du miel certifié sur le marché mondial, à bénéficier des échanges extérieurs et à accroître le produit intérieur brut à travers l'utilisation durable du miombo. (Guni Kokwe, ZAPE)
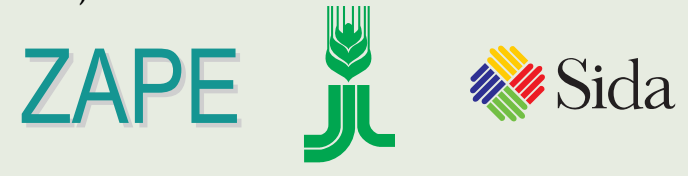

\section{Le baobab dépassant le coton comme la principale source de revenu}

Chapeto habite dans le quartier reculé de Rushinga au nord-est du Zimbabwé, un endroit chaud, sec et poussiéreux où il faut vraiment se battre pour survivre. Chapeto gère une société dénommée "Les Huiles Créatives" qui produit de l'huile à partir des grains de baobab. Il y a trois ans qu'il a investi dans des activités d'extraction de l'huile, et aujourd'hui il gère une petite unité de traitement d'huile dans le village de Chimhanda.

Sa société "les Huiles Créatives" achète régulièrement six tonnes de grains de baobab par mois auprès de soixante producteurs ruraux à Rushinga. Ces producteurs ne cachent pas leur satisfaction parce qu'ils peuvent gagner jusqu'à 180 dollars dans une saison en doublant presque leur revenu par rapport au coton qui est la culture de rente de base. Chapeto aussi est content. Auparavant, il gérait une petite épicerie qui lui procurait un chiffre d'affaires annuel de 4000 dollars US. Aujourd'hui, sa société produit plus de 360 litres d'huile par mois qui lui rapportent près de 9000 dollars US dans une saison.

La réussite de Chapeto est largement dûe à son implication dans "Phyto Trade Africa, la "Southern African Natural Products Trade Association" (L'association des commerçants des produits naturels de l'Afrique Australe). En 2003, Phyto Trade Africa a signé un accord de partenariat avec Aldivia S.A, une société française qui se spécialise dans la production des dérivés des huiles de plantes naturelles qui sont vendus comme des ingrédients de produits de beauté. Aldivia achète l'huile des grains de baobab auprès de Chapeto qui est ensuite traitée et vendue à la société multinationale Bergasol qui à son tour l'incorpore dans une nouvelle crème ou lotion protectrice de la peau. Chapeto n'aurait pas pu réaliser cela seul, mais à travers Phyto Trade Africa, son huile peut maintenant atteindre

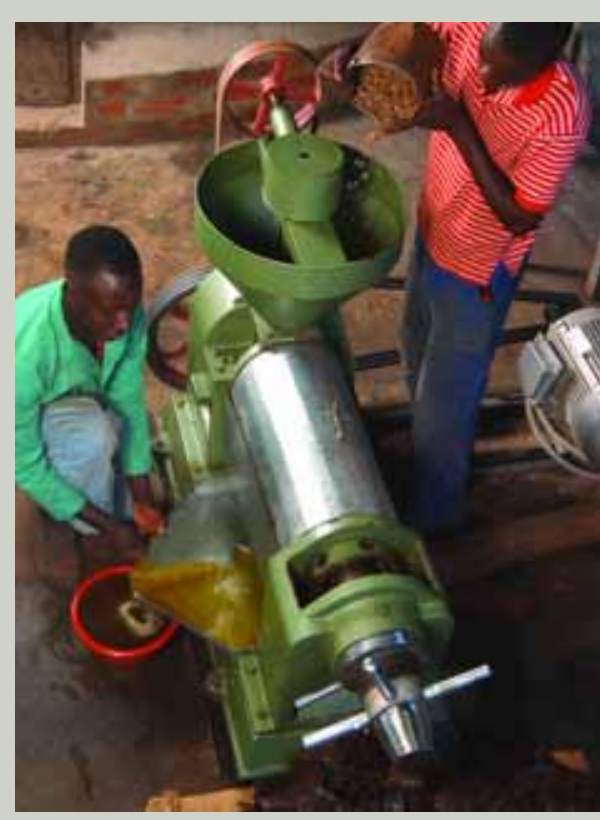

Production d'huile de baobab Photo par Gus le Breton

des consommateurs à travers toute l'Europe et aider à combattre le cancer de la peau.

Il existe de nombreux produits comme l'huile du baobab qui proviennent d'espèces de plantes locales en Afrique rurale ayant des potentialités commerciales significatives. Cependant, pour de petits producteurs tels que Chapeto, il existe de grandes barrières qui empêchent le développement de la commercialisation de ces produits. Mais, en regroupant les ressources avec les autres producteurs à travers l'Afrique Australe et en créant une association commerciale puissante pour représenter ses intérêts, Chapeto a réussi à surmonter ces barrières. (Gus le Breton, Phyto Trade)
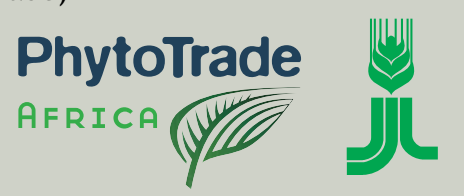
L'utilisation des marchés locaux en Afrique du Sud pour assurer les moyens de subsistance en vue de faire face au VIH/SIDA et au chômage croissant

Le développement de grands marchés extérieurs de produits forestiers constitue une solution pour augmenter les moyens de subsistance des populations (tel que dans les cas Zambiens et Zimbabwéens présentés ci-dessus).

L'autre option consiste à extendre les marchés locaux. Par exemple, à Bushbuckridge, en Afrique du Sud, une variété de produits forestiers localement commercialisés assurent les moyens de subsistance d'une population croissante. Considérez les balais traditionnellement fabriqués à la main avec des herbes sauvages et des espèces d'arbustes qui sont largement utilisés par les ménages : à Bushbuckridge seul qui couvre une superficie de $1800 \mathrm{~km}^{2}$, il y a environ 390000 balais qui sont vendus chaque année.

"Depuis que j'ai commencé à vendre les balais, mes enfants ne vont plus se coucher en pleurant de faim", répondit une mère veuve ayant trois enfants à sa charge quand on lui posa la question concernant les avantages de la vente des balais traditionnels. Pour ces femmes, faire le commerce signifie la différence entre la misère ou la dépendance des parents et des voisins pour leur soutien et l'indépendance ainsi que la capacité à acheter la nourriture et à payer les frais de scolarité. L'analyse du profil des productrices montre que ces gens appartiennent aux couches les plus pauvres et les plus marginalisés de Bushbuckridge. Beaucoup de ménages sont dirigés par des femmes dont la plupart sont des vieilles et des veuves. Beaucoup d'entre elles sont illettrées ou ont reçu une petite instruction. Elles ont peu ou aucune autre source de revenu financier (habituellement il n'y a qu'une allocation sociale des enfants ou une pension) ; le revenu total du ménage est bien en dessous du seuil de pauvreté pour tous les ménages.

Beaucoup de productrices ont dit qu'elles ont commencé à fabriquer et à vendre les balais après qu'elles se sont retrouvées seules pour s'occuper des petits enfants suite à la mort des mères de ces enfants souvent du VIH/SIDA. Même quand elles ont d'autres sources de revenu (telles qu'une pension), cela ne suffit pas à soutenir ces nouveaux dépendants. D'autres productrices se sont engagées dans ce commerce après le décès du soutien de la famille ou après la réduction des dépenses quand leurs maris ne pouvaient plus les soutenir ou ne pouvaient pas trouver du travail. Néanmoins, d'autres cherchent à augmenter leurs maigres revenus existants. Elles s'adonnent à des activités dont elles possèdent les compétences et dont le marché existe déjà. "Le commerce du balai constitue un moyen qui nous permet de gagner de l'argent et que nous pouvons apprendre à gérer nous-mêmes", a dit une productrice. Plus du tiers des fabricantes des balais font ce commerce depuis 1-5 ans seulement, ce qui reflète la tendance nationale croissante vers une plus grande implication dans le commerce des ressources naturelles comme une réponse au chômage croissant, aux impacts du VIH/SIDA et à d'autres difficultés économiques.

Les revenus obtenus sont généralement modestes bien que certains entrepreneurs qui commercialisent plus de produits puissent obtenir des revenus beaucoup plus élevés que les taux des salaires locaux. Par exemple, plusieurs femmes qui avaient travaillé comme des femmes de ménage dans des familles locales où les salaires étaient particulièrement bas, ont trouvé qu'elles gagnaient plus avec la vente des balais. Elles pouvaient aussi être plus indépendantes et flexibles. Les bénéfices nets provenant de la vente des balais se situaient entre 7 dollars US et 1680 dollars par an avec une moyenne de plus de 300 dollars US. Même les petits montants peuvent faire la différence car ils permettent de subvenir aux besoins de la famille en période critique. La vente des balais a permis à une des productrices de réaliser des travaux d'aménagement sur sa maison y compris la réfection de la toiture en tuiles.

Des petits changements au niveau des marchés locaux de produits forestiers tels que le renforcement de la sécurité des droits d'accès aux ressources, l'amélioration de l'organisation des producteurs dans les activités de récolte et de commercialisation, l'amélioration du système des transports et moins de bureaucratie pour le marketing, seront bénéfiques aux producteurs. L'accès au micro-crédit permettrait aux gens de supporter les frais de transport pour obtenir plus de matières premières. (Sheona RHODES UNIVERSITY Shackleton, Rhodes University) 


\section{De la poubelle à l'agroforêt durable à Ouagadougou, Burkina Faso}

Dans la banlieue de Ouagadougou, Madame Kaboré et ses amies de l'AMIFOB (Amicale des Forestières du Burkina) ont initié le groupe local de femmes dénommé Nabonswendé. Le nom du groupe - "nous demandons à Dieu" - reflète les conditions épouvantables dans lesquelles vivaient ces femmes. "Nous gagnions notre vie en ramassant du sable et du gravions pour vendre, et ce ramassage se faisait sur une zone qui servait de poubelle c'était un travail dur dans des conditions désagréables qui nous procurait très peu d'argent". La plupart des 42 membres du groupes sont des veuves qui doivent s'occuper de leurs familles.

Grâce à l'aide de l'AMIFOB et de FEM/ONG (Fonds pour l'Environnement Mondial aux ONG), le groupe a créé un jardin à usages multiples dans une des banlieues paurres et peuplées de la capitale. Le succès de l'initiative a permis de remporter deux prix, l'un au niveau national et l'autre, au niveau régional, du Comité Inter Etat Permanent de Lutte contre la Sécheresse dans le Sahel (CILSS).

Elles ont créé un jardin potager, une pépinière ayant une production de 30.000 plants destinés à la vente ou à la plantation dans des zones spécifiques, un arboretum de 3 ha comportant 600 arbres et 75 espèces locales exotiques, y compris des plantes médicinales, des arbres fruitiers, des arbres pour fourrages et des plantes d'ornement. Ces activités agricoles et forestières ont donné l'occasion d'exercer une variété d'autres activités. L'UNESCO a construit un hangar sur le site où se déroulent des séances de formation traitant de thèmes différents tels que la maternité, l'alphabétisation et le séchage de poisson. L'arboretum est de plus en plus utilisé pour enseigner l'utilité des plantes sauvages aux élèves de la ville en matière de nutrition et de soins médicaux, etc. "Nous nous sommes rendu compte que beaucoup d'enfants de la ville n'avaient aucune idée des différents fruits et feuilles sauvages qui sont importants dans les villages, et encore moins des arbres et des arbustes sur lesquels poussent ces fruits et ces feuilles. Beaucoup d'enfants ne peuvent pas reconnaître le karité et ne savent pas que le savon qu'ils utilisent quotidiennement est un produit de cet arbre".

Ces projets contribuent à réduire la pauvreté. La vente des plants et des légumes permet au groupe de générer plus de 3500 dollars par an, ce qui est un montant considérable, comparativement à leur revenu antérieur. Les femmes peuvent maintenant acheter des habits pour leurs familles, envoyer leurs enfants à l'école, s'occuper de leur santé, consommer de la nourriture de meilleure qualité, et elles ont pris plus de conscience de leur environnement local. Le défi actuel aux acteurs de développement est d'envisager de telles initiatives à plus grande échelle et de créer d'autres groupes similaires de manière rentable. (AMIFOB)

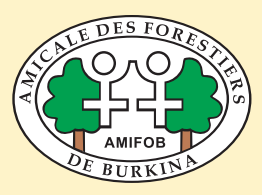

\section{La voie pour aller de l'avant}

Pour atteindre leurs objectifs, les stratégies de réduction de la pauvreté en Afrique doivent tenir compte du rôle central que jouent les forêts en soutenant les moyens de subsistance ruraux notamment des communautés pauvres et marginalisées. Les Documents de Stratégie de Réduction de la Pauvreté (DRSP) sont des instruments clés que les gouvernements des pays les moins développés doivent considérer pour définir les priorités politiques et budgétaires. Les Programmes Nationales des Forêts (PNF) sont également importants pour les forêts. Cependant, dans les deux cas, la contribution des forêts naturelles au soutien des pauvres est négligée et sous-estimée. Les interventions locales basées sur les produits forestiers dont la promotion est faite avec succès par des agences non gouvernementales, doivent pourtant être développées pour couvrir plus de gens sur des superficies beaucoup plus grandes. Les stratégies recommandées pour atteindre ce but comprennent la diffusion des informations clé, le plaidoyer, l'habilitation et l'engagement dans les processus en matière de politique. Les forêts et la foresterie doivent être reconsidérées dans les programmes nationaux et mondiaux.

Ce document a été préparé par AMIFOB, Bruce Campbell (b.campbell@cgiar.org), Peter Frost, Guni Kokwe, Gus le Breton, Sheona Shackleton et Daniel Tiveau. Les opinions exprimées dans cette publication sont celles des auteurs et pas nécessairement celles de CIFOR. L’Asdi a généreusement financé le travail de CIFOR sur "Stimuler le dialogue politique sur l'aménagement durable des forêts sèches en Afrique.

Programme forêts et moyens d'existence, CIFOR

Bureau: Jalan CIFOR, Situ Gede, Sindang Barang, Bogor Barat 16680, Indonesia. Adresse postale: P.O. Box. 6596 JKPWB, Jakarta 10065, Indonesia
Tel: +62(251) 622622 Fax: +62(251) 622100

Mail: cifor@cgiar.org Site web: www.cifor.cgiar.org

Photos de couverture par: Peter Frost et Bruce Campbell 